\title{
Min-Min Times in Peer-to-Peer File Sharing Networks
}

\author{
G. Matthew Ezovski, Animashree Anandkumar, and A. Kevin Tang \\ School of Electrical and Computer Engineering \\ Cornell University \\ Ithaca, NY 14853 \\ \{gme8, aa332, at422\} @cornell. edu
}

\author{
Lachlan L.H. Andrew \\ Computer Science \\ California Institute of Technology \\ Pasadena, CA 91125 \\ lachlanecaltech.edu
}

\begin{abstract}
Peer-to-peer (P2P) file distribution is a scalable way to disseminate content to a wide audience. This paper presents an algorithm by which download times are sequentially minimized; that is, the first peer's download time is minimized, and subsequent peers' times are minimized conditional on their predecessors' times being minimized. This objective gives robustness to the file distribution in the case that the network may be partitioned. It is also an important step towards the natural objective of minimizing the average download time, which is made challenging by the combinatorial structure of the problem. This optimality result not only provides fundamental insight to scheduling in such P2P systems, but also can serve as a benchmark to evaluate practical algorithms and illustrate the scalability of P2P networks.
\end{abstract}

\section{INTRODUCTION}

Peer-to-peer (P2P) networking utilities are among the most frequently used applications on the Internet and have often been observed to consume large fractions of available Internet bandwidth. In fact, studies [19], [20] have shown that upwards of $45 \%$ of Internet traffic can be attributed to P2P applications. They have also generated a great deal of research activity in the last couple of years; see e.g., [3]-[6], [8], [12], [20], [22], [24] and the references therein.

The fundamental advantage of peer-to-peer architectures compared with classic client-server architectures is their scalability. As every peer is both a client and a server at the same time, a P2P network can potentially distribute data to a large number of peers in a much shorter period of time. This paper considers a classical situation, in which a file is to be distributed as quickly as possible to a known set of peers. This can be used as a basic model for many scenarios such as distributing a software patch to an existing subscriber base. It is also a standard model used to illustrate the scalability of P2P networks [9], in which one can calculate the amount of time needed to distribute a file of certain size to all peers under both $\mathrm{P} 2 \mathrm{P}$ and client-server architectures. The calculation is typically done using the last finish time metric, which is defined to be the time when the last peer gets the complete file. Another natural fundamental metric is average finish time, which is the sum of finish times of all peers divided by the number of peers. However, minimizing it brings significantly more analytical challenges and this paper is devoted to the intermediate step of finding an explicit scheduling procedure to sequentially minimize the download times.
Several papers have explored performance of P2P networks [1], [8], [13], [15]-[17], [21], [23], [25]. Several of these deal with optimal scheduling algorithms. For example, Mundinger et al. [16], [17] characterize the problem of file sharing in networks with heterogeneous upload capacities and discrete file divisions. They also explore initial results for cases where the file to be shared can be divided into infinitely small pieces. Another example is [8] which discusses optimal strategies for file distribution when multiple classes of service exist. Recently, Mehyar et al. [15] extended Mundinger's upload-constrained result and look at average finish time problems. They provide solutions for all cases in which the number of nodes is three or less, as well as solutions to a special class of larger cases. Building upon all this work while identifying new inductive structures and using new techniques such as water-filling, this paper provides a complete explicit algorithm to minimize average finish time with an arbitrary number of peers.

The main difficulty of the design of optimal filedistribution algorithms is the need to keep track of data identity. In other words, a node must receive a whole file, rather than just an amount of data equal to the file size which could include much duplication. This complicates the problem of how a node should choose to send a piece of data from "who most needs this amount of data?" to "who most needs this particular piece of data?" Ignoring this constraint significantly reduces the complexity of the problem [18] but results in unrealistic results. In general, how the overall network benefits from the decision to send a particular piece of data to a particular node depends on the optimality criterion, as well as the physical constraints of the nodes involved.

This paper is a step towards addressing the problem of designing explicit file dissemination scheduling algorithms which provably minimize average finish time. To overcome the above mentioned difficulty, our overall strategy is to use an intermediate step by introducing another concept (min-min time), which has an inherent inductive structure that facilitates algorithm design. This sub-problem is of independent interest when there is a chance that the network will be partitioned by network failures; by minimizing the time until another node has an entire copy of the file, this schedule improves the probability that all nodes will eventually be able to recieve the complete file [11].

The paper is organized as follows. Section II reviews the 
solution that achieves the optimal last finish time and then formulates the min-min and average finish time problems. After that, we present the main result in section III, where an explicit solution to achieve the optimal min-min times is provided, along with a water-filling interpretation. We conclude in section IV and discuss some possible interesting extensions.

\section{Problem Formulation}

\section{A. Model and notation}

Consider a single node, referred to as the server, which needs to distribute a file of size $|F|$ to $N$ peer nodes. The system is assumed to be churn-free, in that peers neither arrive nor leave. We assume that there are no topological constraints; each node, including the server, can communicate with each other node with no bottlenecks other than the nodes' upload constraints. Finally, the file can be broken into infinitesimally small pieces; thus, there is no forwarding delay, and a node can immediately relay what it receives to another node.

This paper uses the following notation:

- $|F|$ : size of the file

- $F_{i}(t)$ : portion of the file that peer $i$ has at time $t$

- $\left|F_{i}(t)\right|$ : size of that portion

- $N$ : total number of peer nodes (not including the server)

- $C_{0}$ : server upload capacity

- $C_{i}$ : node $i$ upload capacity $C_{1} \geq C_{2} \geq \ldots \geq C_{N}$

- $C=C_{0}+\sum_{i=1}^{N} C_{i}$ : total system capacity

- $R_{i j}(t, t+\tau)$ : data sent from node $i$ to node $j$ in the interval $(t, t+\tau)$.

- $r_{i j}(t)=\frac{d}{d t}\left|R_{i j}(0, t)\right|:$ rate at which node $i$ sends to node $j$ at time $t$

- Finish time $t_{i}$ for peer $i$ : the smallest $t$ with $\left|F_{i}(t)\right|=$ $|F|$

- $|F| / C_{0}$ - bottleneck time: the time it takes for one node to directly receive the entire file from the server, and a lower bound on the time for all nodes to receive the file

We consider an upload-constrained scenario in which each node can receive information with unlimited data rate, but the

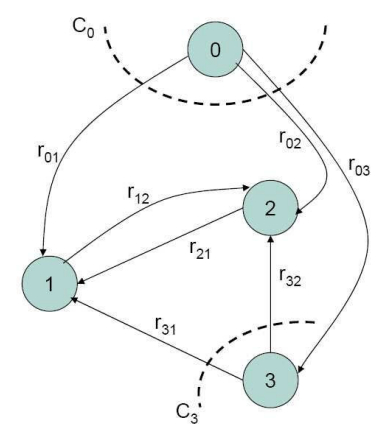

Fig. 1. A diagram showing the constraints on communication between nodes in a 3-node plus server configuration. The dashed lines represent the sum rate constraints $\sum_{j=0}^{N} r_{i j}(t) \leq C_{i} \forall i$. sum rate of any uploads from each node must be no greater than that node's given upload capacity. Mathematically,

$$
\sum_{j=1}^{N} r_{i j}(t) \leq C_{i} \quad \forall i, t .
$$

The "data identity" constraint can now be expressed as

- $R_{i j}(t, t+\tau) \subseteq F_{i}(t+\tau)$ (received data constraint; can only send data already received)

- $R_{i j}(t, t+\tau) \cap F_{j}(t)=\emptyset$ (only receive new data)

- $R_{i j}(t, t+\tau) \cap R_{k j}(t, t+\tau)=\emptyset \quad \forall i \neq k$ (only receive non-duplicate data)

- $r_{i i}(t)=0$ (a node can't send data to itself)

- $F_{j}(t)=\bigcup_{i=0}^{N} R_{i j}(0, t)$, whence

- $\frac{d}{d t}\left|F_{j}(t)\right|=\sum_{i=0}^{N} r_{i j}(t) \quad \forall j, t$.

\section{B. Average Finish Time}

We first briefly review the problem of minimizing the last finish time (the time for all nodes in the network to receive the entire file). Clearly, this time, $T_{L}^{*}$, can't be less than $|F| / C_{0}$, which is the time it takes for the server to send the file to one recipient, or less than the time it would take to share the file with all nodes if every node in the network was fully utilized for all time, $N|F| / C$. Formally,

$$
T_{L}^{*} \geq \max \left(|F| / C_{0}, N|F| / C\right)
$$

Mundinger et al. [16] show that this lower bound is tight by looking at the following two possibilities.

1) Case 1 - Fast Server: When $C_{0} \geq \sum_{i=1}^{N} C_{i} /(N-1)$, each peer is assigned server capacity of rate $C_{i} /(N-1)$, and each peer can then re-upload to the remaining $N-1$ peers at rate $C_{i} /(N-1)$. The excess capacity is shared equally. This results in each peer receiving total capacity $C / N$ on the time interval $\left(0, T_{L}^{*}\right)$.

2) Case 2 - Slow Server: When $C_{0} \leq \sum_{i=1}^{N} C_{i} /(N-1)$, the server can allocate to each peer $i$ an upload rate of

$$
\frac{C_{i} C_{0}}{\sum_{j=1}^{N} C_{j}}
$$

which does not exceed that peer's upload capacity. Each node can forward on what it receives to every other peer; thus, each peer effectively receives at rate $C_{0}$ from the server.

It turns out that forcing all the nodes to finish receiving the file at $T_{L}^{*}$ might artificially limit the performance of the network by other metrics. In other words, by allowing small increases in $T_{L}>T_{L}^{*}$, we can potentially substantially decrease the average finish time, $T_{A}$, and thus improve the overall performance of the network. This is illustrated with the following simple numerical example.

Example 1: Potential improvement over minimizing last finish time.

Let $N=4$, with $C_{0}=12, C_{1}=6, C_{2}=4, C_{3}=2, C_{4}=$ 1 , and $|F|=144$. We calculate the optimal last finish time $T_{L}^{*}$ and the optimal average finish time, $T_{A}$. The results are summarized in Fig. 2. By allowing a very small upward shift in finish time $t_{4}$, substantial improvements in other finish times can be achieved. For example, with the selected set 
of upload capacities and specified file size, an average finish time decrease of $28.9 \%$ corresponds to a $0.91 \%$ increase in last finish time.

It is now clear that the average finish time is an important performance metric. Formally, we have

$$
T_{A}=\frac{\sum_{i=1}^{N} t_{i}}{N} .
$$

In general, to minimize the average finish time, we want to maximize the rate at which information is exchanged in the network for all times, and attempt to minimize the finish times of nodes with high capacity as quickly as possible. However, due to the combinatorial structure of the problem and especially the data identity constraint, it is hard even to write down the optimization problem for general case. The following example illustrates this difficulty with a very simple 2-peer network.

\section{Example 2: Direct minimizing average finish time for a two-} peer network.

Consider the 2-peer case, we can set up a linear program which optimizes the average finish time by adjusting the sizes of the blocks of data the nodes send to each other in each time interval within the constraints of the problem.

$$
\begin{aligned}
\min _{R_{01}, R_{02}, R_{12}} & t_{1}+t_{2} \\
\text { subject to } & t_{1}=\left|R_{01}\left(0, t_{1}\right)\right| /\left(\lambda C_{0}\right) \\
& t_{2}=t_{1}+\left(\left|R_{01}\left(0, t_{1}\right)\right|-\left|R_{12}\left(0, t_{1}\right)\right|\right. \\
& \quad-\frac{\left.\left|R_{01}\left(0, t_{1}\right) \cap R_{02}\left(0, t_{1}\right)\right|\right)}{\left(C_{1}+C_{0}\right)} \\
& \lambda=\left|R_{01}\left(0, t_{1}\right)\right| /\left(\left|R_{01}\left(0, t_{1}\right)\right|+\left|R_{02}\left(0, t_{1}\right)\right|\right) \\
& \left|R_{01}\left(0, t_{1}\right) \cup R_{02}\left(0, t_{1}\right)\right|=|F| \\
& \left|R_{21}\left(0, t_{1}\right)\right| \leq C_{2} t_{1} \\
& \left|R_{12}\left(0, t_{1}\right)\right| \leq C_{1} t_{1} \\
& \left|R_{01}\left(0, t_{1}\right)\right|+\left|R_{02}\left(0, t_{1}\right)\right|=C_{0} t_{1} \\
& \left|R_{21}\left(0, t_{1}\right)\right|=\left|R_{02}\left(0, t_{1}\right) \backslash R_{01}\left(0, t_{1}\right)\right| \\
& \left|R_{12}\left(0, t_{1}\right)\right| \leq\left|R_{01}\left(0, t_{1}\right)\right|
\end{aligned}
$$

Here the data identity constraint forces us to keep track of the sizes of many distinct pieces of data even when $N=2$

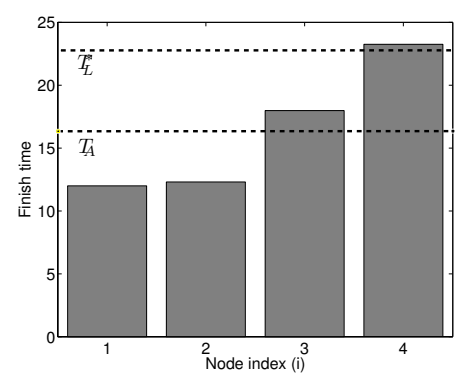

Fig. 2. Results for the $N=4$ case, with $C_{0}=12, C_{1}=6, C_{2}=4$, $C_{3}=2, C_{4}=1$, and $|F|=144 . T_{A}$ is the associated average finish time, and $T_{L}^{*}$ is the optimal last finish time. (the last six constraints in the above optimization). In general, similar optimizations can be written for larger $N$, but the number of variables and constraints grows exponentially with the size of the problem. This difficulty motivates us to look for inductive structures which allows us not to optimize all data pieces at the same time. The min-min times that will be introduced in section II-C serve this role.

\section{Min-Min Times}

The min-min time sequentially minimizes the individual finish times. Besides its relation to the optimal average finish time, it is also of independent interest, since minimizing the completion times of early flows improves the robustness to disconnection of the network [11].

Formally, let $t_{i}^{s}$ be the finish time of peer $i$ under rate scheme $s$.

- Let $S_{1}$ be the set of schemes which minimize time $t_{1}$.

- Let $S_{i+1}$ be the set of schemes which minimize the $i+1$ st finish time, given that all previous finish times are minimized.

A scheme in $s \in S_{N}$ is said to achieve min-min times, and the times $t_{i}^{s}$ are called the min-min times.

The inductive structure imposed by sequential minimization allows us to find an explicit schedule to achieve minmin times. This will be shown in section III. Before delving into our main results, we introduce the useful concept of multiplicity [15], which will be used to classify problems. Define multiplicity, $M$, as the maximum number of nodes which can receive a file with size $|F|$ in bottleneck time $|F| / C_{0}$. The following lemma is proved in [14].

Lemma 1: Let $M$ be the largest value of $K$ such that there exists a schedule with

$$
F_{i}\left(\frac{|F|}{C_{0}}\right)=F, \quad \forall i \leq K .
$$

Then $M$ is the largest integer such that

$$
C_{0} \leq \sum_{i=1}^{M} \frac{C_{i}}{M-1}+\sum_{M+1}^{N} \frac{C_{i}}{M} .
$$

\section{Scheduling to Achieve Min-Min Times}

When the multiplicity $M=N$, all nodes can finish by $|F| / C_{0}$ using the schedule reviewed in section II-B. We now study optimal schedules for the remaining cases $(M<N)$.

The main difficulty in achieving min-min times is when we try to minimize $t_{i}$, how to use the extra capacities of some peers. It will be shown that they only need to minimize $t_{i+1}$. In other words, scheduling for more than one step ahead is not useful. Another difficulty is how to schedule all peers to minimize $t_{i}$ given they all have different capacities $C_{i}$. A "water filling" technique will be used to decide optimal scheduling for all peers. The potential contributions of finished nodes to the next finishing node can be thought of as "water", and the data scheduled to be shared by other nodes forms an uneven floor.

This is illustrated in Fig. 3(a). During the interval $\left(t_{i-1}, t_{i}\right)$, the $j$ th column has width $C_{j}$, and area $F_{j}\left(t_{1}\right) \backslash$ 
$F_{i}\left(t_{i-1}\right)$. Thus, the depth is the minimum time it would take for node $j$ to upload all of the data it could to node $i$.

Note that the sets $F_{j}\left(t_{1}\right) \backslash F_{i}\left(t_{i-1}\right)$ are disjoint for $j>$ $i$. (This will be guaranteed by our scheduling algorithm.) Thus, the region in columns $j>i$ is exactly the data which must be transmitted to node $i$ in the interval $\left(t_{i-1}, t_{i}\right)$, and the question is who should transmit what to minimize this interval. If the server and completed nodes did not send any further data to node $i$, the maximum depth is the minimum possible value of $t_{i}-t_{i-1}$ (column $\mathrm{N}$ in Fig. 3). The optimal way is to let nodes $0 \leq j<i$ send the shaded data in Fig. 3(b), equalizing the finish times $t_{i}-t_{i-1}=\left|F_{j}\left(t_{1}\right)\right|$ $F_{i}\left(t_{i-1}\right) \mid / C_{j}$.

The only remaining question is what node $i$ should do when others are uploading data to it. Due to the "data identity" constraint, $r_{i i}(t)=0$, and therefore it cannot transfer data to itself. The optimal way is to use node $i$ 's capacity to send data to $i+1$. The specific data to be sent will be determined by "helium-filling" for the following time interval, $\left(t_{i}, t_{i+1}\right)$ as follows: Data $U_{i j}$, sent at rate $\gamma_{i} j$, is chosen such that it would have been in column $j$ at time $t_{i}$ had it not been sent to node $i+1$ in interval $\left(t_{i-1}, t_{i}\right)$, but it instead "comes off the top" of the columns, in proportion to their capacities (Fig. 3(b)). (Note that $U_{i 0}$ corresponds to data which would have been sent by the server on the interval $\left(t_{i}, t_{i+1}\right)$, and thus is represented by "water," but is instead sent by node $i$ on the previous interval.) In later proofs, we will provide specialized water-filling figures for different cases (Figures 4 and 5).

The actual scheduling algorithm is stated in Algorithm 1. It uses $C_{0}^{*}$, which is an upper bound on the range of $C_{0}$, for a particular given multiplicity $M$, for which exactly one set of optimal values $F_{i}\left(|F| / C_{0}\right), \forall i>M+1$, is able to achieve first $M+1$ min-min times. Formally, it is the solution to

$$
\begin{aligned}
& \frac{M\left(C_{0}^{*}-\frac{C_{M+1}}{M}-\sum_{i=1}^{M} \frac{C_{i}}{M-1}\right)+C_{0}^{*}}{C_{0}^{*}+\sum_{i=1}^{M} C_{i}} \\
& =\frac{(M+1)\left(C_{0}^{*}-\frac{C_{M+1}}{M}-\sum_{i=1}^{M} \frac{C_{i}}{M-1}\right)}{\sum_{i=M+2}^{N} C_{i}} .
\end{aligned}
$$

When $C_{0}>C_{0}^{*}$, there could be multiple sets of $F_{i}\left(|F| / C_{0}\right)$, $\forall i>M+1$ that all achieve the first $M+1$ min-min times. Then Algorithm 1 also uses the following linear program to select the only set of $F_{i}\left(|F| / C_{0}\right)$ values which allows all min-min times to be achieved.

$$
\max \sum_{i=M+2}^{N}(N-i) \lambda_{i}
$$

s.t.

$$
\begin{gathered}
\frac{C_{i}}{M+1}<\lambda_{i} \leq \frac{C_{i}}{M} \quad \forall i \geq M+2 \\
\sum_{i=M+2}^{N} \lambda_{i}=C_{0}-\frac{C_{M+1}}{M}-\sum_{i=1}^{M} \frac{C_{i}}{M-1} \\
\frac{(M+1) \lambda_{i}-C_{i}}{C_{i}} \geq \\
\frac{\frac{1}{M-1} \sum_{i=1}^{M} C_{i}+(M+1) \sum_{i=M+2}^{N} \lambda_{i}-\sum_{i=M+2}^{N} C_{i}}{C-C_{M+1}}
\end{gathered}
$$

The following theorem characterizes Algorithm 1.

Theorem 1: Algorithm 1 achieves min-min times.

Proof: We state the proof for $M=1$ here; the proof for $1<M<N$ can be found in Appendix I.

Note first that Algorithm 1 is feasible. In particular, until time $t_{i}$, all nodes $j>i$ have disjoint data, while nodes $j<i$ have all data. Similarly, $U_{i, j}$ can be forwarded by $i$ as it is received from node $j$, since $\gamma_{i, j} \leq C_{j}$, allowing the three claimed conditions to be satisfied.

It remains to establish optimality. Let $\underline{t}_{i}$ denote the minmin finish time of node $i$. The proof of optimality first establishes lower bounds on $\underline{t}_{1}$ and $\underline{t}_{2}$, and shows that Algorithm 1 achieves those times, and that the $\lambda_{i}$ are the unique values which can achieve that. It then inductively shows that subsequent times are minimized.

Let $C^{\prime}=\sum_{i=3}^{N} C_{i}$. This can be thought of as the capacity of a "virtual node" consisting of nodes $3, \ldots, N$. As in [15], the amount of information that can go into nodes 1 and 2 on $\left(0, t_{2}\right)$ is bounded above as

$$
F_{1}\left(t_{2}\right)+F_{2}\left(t_{2}\right) \leq\left(C_{0}+C_{1}\right) t_{2}+C_{2} t_{1}+\frac{C^{\prime}}{2} t_{2} .
$$

The first terms shows that the server and node 1 can contribute on the whole time interval. The second term reflects node 2's transmission to node 1 on $\left(0, t_{1}\right)$; on $\left(t_{1}, t_{2}\right)$, it cannot contribute, since it cannot upload to itself, and on $\left(t_{1}, t_{2}\right)$ node 1 has already received the whole file. The term $t_{2} C^{\prime} / 2$ arises as follows. Node $i \geq 3$ can send information

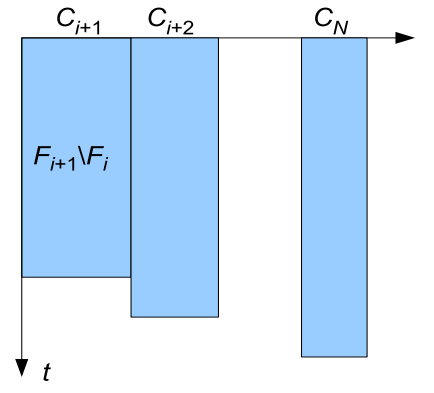

(a)

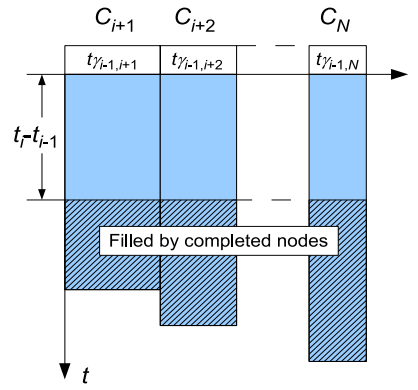

(b)
Fig. 3. Water filling. The width of column $j$ is capacity $C_{j}$, and the depth is the time to transmit $F_{j}\left(t_{1}\right) \backslash F_{i}\left(t_{i-1}\right)$ at rate $C_{j}$. In (a), node $N$ takes longer to transmit its information. In (b), the server has waterfilled, decreasing the time for all to complete transmission to node $i$, and allowing full utilization for the interval. The helium-filling by $\left(t_{i}-t_{i-1}\right) \gamma_{i j}$ in interval $\left(t_{i-1}, t_{i}\right)$ reduces the heights of all columns equally. 


$$
\begin{aligned}
& \overline{\text { Algorithm 1 Optimal scheduling to achieve min-min times }} \\
& \hline \text { If } \mathrm{M}=1 \\
& \text { - On }\left(0, t_{1}\right), \text { let } r_{0 i}=\lambda_{i}, r_{i 1}=\min \left(\lambda_{i}, C_{i}\right), r_{i 2}=C_{i}- \\
& \min \left(\lambda_{i}, C_{i}\right) \text {, where } \lambda_{i} \text { satisfy } \\
& \sum_{i=1}^{N} \lambda_{i}=C_{0} \quad \lambda_{2}=C_{2} \quad \frac{2 \lambda_{i}}{C_{i}}=\frac{C_{0}+\lambda_{1}}{C_{0}+C_{1}}, \forall i>2 .
\end{aligned}
$$

- On $\left(t_{i-1}, t_{i}\right), 2 \leq i<N$ :

$r_{j i}(t)=C_{j} \forall j \neq i$, with $R_{j i}\left(t_{i-1}, t_{i}\right) \cap$ $R_{k i}\left(t_{i-1}, t_{i}\right)=\emptyset$ and $\left(R_{j i}\left(t_{i-1}, t_{i}\right) \cup R_{k i}\left(t_{i-1}, t_{i}\right)\right) \cap$ $F_{i}\left(t_{i-1}\right)=\emptyset$ for all $k \neq j$. Node $i$ sends data $U_{i j}$ (helium) to node $i+1$ with $r_{i, i+1}(t)=C_{i}$ such that

1) $U_{i j} \cap U_{i k}=\emptyset$ for all $k \neq j$ (data is disjoint)

2) $U_{i j} \in F_{j}\left(t_{i-1}\right)$ (data is held at $t_{i-1}$ by node $j$ )

3) for all $j \geq 0, j \neq i+1$,

$$
\frac{\left|U_{i j}\right|}{t_{i}-t_{i-1}}=\gamma_{i j}=\frac{C_{j}}{\left(\sum_{k=0, k \neq i+1}^{N} C_{k}\right)} C_{i} .
$$

Else

- If $M=N-1$ or $C_{0} \leq C_{0}^{*}$, given by (4),

Then let $\lambda_{i}$ solve

$$
\begin{gathered}
\sum_{i=1}^{N} \lambda_{i}=C_{0} \\
\frac{\lambda_{i}}{C_{i}}= \begin{cases}\lambda_{1} / C_{1} & \text { if } i \leq M \\
1 / M & \text { if } i=M+1 \\
\lambda_{M+2} / C_{M+2} & \text { if } i \geq M+2\end{cases} \\
\frac{\lambda_{M+2}}{C_{M+2}}=\frac{M+\sum_{i=1}^{M} \lambda_{i}+C_{0}}{(M+1) \sum_{i=0}^{M} C_{i}}
\end{gathered}
$$

Else let $\lambda_{i}=C_{i} /(M-1), \forall i \leq M$, and let $\lambda_{i}$ for $i \geq M+2$ satisfy the LP (5).

\section{EndIf}

- On $\left(0, t_{M}\right)$ :

$r_{0 i}=\lambda_{i}, \forall i ; r_{i j}(t)=\lambda_{i}$ for $j \leq M, j \neq i ; r_{i j}(t)=0$ for $j>M+1 ; r_{i, M+1}(t)=C_{i}-\sum_{j \neq M+1} r_{i j}(t)$.

- On $\left(t_{i-1}, t_{i}\right)$ for $M+1 \leq i<N$ : $r_{j i}(t)=C_{j}$ for $j \neq i$, such that

$$
\left|R_{0 i}\left(t_{i-1}, t_{i}\right) \cap F_{j}\left(t_{i-1}\right)\right|=\mu_{j i}\left(t_{i}-t_{i-1}\right),
$$

for $j<i$, where

$$
\begin{aligned}
\mu_{j, M+1} & =\frac{C_{0}}{\left(\sum_{k=1}^{N} C_{k}\right)-C_{M+1}} C_{j}, \quad \forall j \neq M+1, \\
\mu_{j, i} & =\lambda_{i} t_{1}-C_{i} \frac{\left(C_{0}-\lambda_{i}\right) t_{1}}{C_{0}+C-C_{i}}, \quad \forall j \neq i \neq M+1 .
\end{aligned}
$$

Also $r_{i, i+1}(t)=C_{i}$, such that $\mid R_{i, i+1}\left(t_{i-1}, t_{i}\right) \cap$ $F_{j}\left(t_{i-1}\right) \mid=\gamma_{j i}$ where

$$
\gamma_{j i}=\frac{C_{i} C_{j}}{\left(\sum_{k=1}^{N} C_{k}\right)-C_{i+1}}, \quad \forall j \neq i+1
$$

EndIf

On $\left(t_{N-1}, t_{N}\right), r_{i N}(t)=C_{i}$ for $i<N$, and $r_{i k}(t)=0 \quad \forall k$.

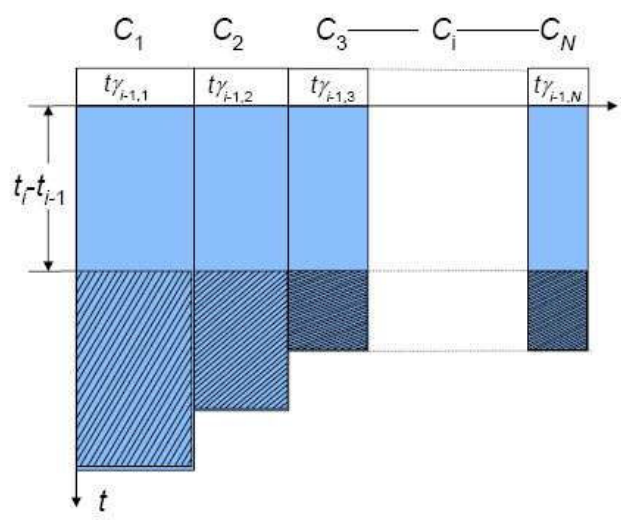

Fig. 4. A visual depiction of the waterfilling argument for the case when $M=1$. Note that the bottoms of columns $M+2, \ldots, N$ are level.

which it has received up to time $t_{2}$ to both nodes 1 and 2, but it cannot exceed its own upload capacity, and cannot upload to $t_{1}$ data which it does not have until $t_{1}$. Thus, its uploads to 1 and 2 are bounded above by $\min \left\{C_{i} t_{2}, F_{i}\left(t_{1}\right)+F_{i}\left(t_{2}\right)\right\}$. However, the data obtained by node $i$ from the server comes at the expense of data that the server could have sent to node 1 or 2 directly, giving a net contribution of

$$
\min \left\{C_{i} t_{2}, F_{i}\left(t_{1}\right)+F_{i}\left(t_{2}\right)\right\}-F_{i}\left(t_{2}\right) .
$$

Note that

$$
\min \left\{C_{i} t_{2}, F_{i}\left(t_{1}\right)+F_{i}\left(t_{2}\right)\right\} \leq \frac{C_{i} t_{2}+2 F_{i}\left(t_{2}\right)}{2}
$$

with equality only if

$$
2 F_{i}\left(t_{1}\right)=2 F_{i}\left(t_{2}\right)=C_{i} t_{2}
$$

Substituting (9) into (8) and summing over $i \geq 3$ gives $C^{\prime} T_{2} / 2$, establishing (7).

A lower bound on $\underline{t}_{2}$ results from substituting $F_{1}\left(t_{2}\right)+$ $F_{2}\left(t_{2}\right)=2 F$ into (7), and substituting the known value $\underline{t}_{1}=$ $|F| / C_{0}$, giving

$$
\underline{t}_{2} \geq \frac{2|F|-C_{2}|F| / C_{0}}{C_{0}+C_{1}+C^{\prime} / 2}
$$

This is achieved by Algorithm 1.

To see that the choice of $\lambda_{i}$ is the only one which achieves $\underline{t}_{2}$, note that (10) is a necessary condition for all $i \geq 3$. Dividing by $C_{i} t_{1}$ and substituting $\lambda_{i}=\left|F_{i}\left(t_{1}\right)\right| / t_{1}$ gives

$$
\frac{2 \lambda_{i}}{C_{i}}=\frac{\underline{t}_{2}}{\underline{t}_{1}}
$$

for all $i \geq 3$. Similarly, the data known only to node 1 and the server at $t_{1}$, of which there is an amount $\left(\lambda_{1}-C_{1}\right) t_{1}$, must also be delivered at rate $C_{1}+C_{0}$ in time $t_{2}-t_{1}$. Dividing by $t_{1}$ and adding 1 gives

$$
\frac{\lambda_{1}+C_{0}}{C_{0}+C_{1}}=\frac{\underline{t}_{2}}{\underline{t}_{1}} .
$$


Combining (12) and (13) shows that $\lambda_{i}, i>2$, must satisfy (6) to achieve $\underline{t}_{2}$. Thus, Algorithm 1 achieves $\underline{t}_{1}$ and $\underline{t}_{2}$, and (6) are necessary for any scheme which does.

Given that (6) must hold in order to achieve $\underline{t}_{1}$ and $\underline{t}_{2}$, it can be shown by induction on $i$ that: (a) node $i$ receives no data in the interval $\left(t_{1}, t_{i-2}\right)$, and (b) $\underline{t}_{i}$ is tightly bounded below by

$$
\underline{t}_{i} \geq \frac{|F|-\lambda_{i} \underline{t}_{1}-C_{i-1}\left(\underline{t}_{i-1}-\underline{t}_{i-2}\right)}{C-C_{i}}+\underline{t}_{i-1} .
$$

The term $\lambda_{i} \underline{t}_{1}$ is the amount of data received by node $i$ from the server during the first interval, $\left(0, \underline{t}_{1}\right)$, and the term $C_{i-1}\left(\underline{t}_{i-1}-\underline{t}_{i-2}\right)$ is the data received from node $i-1$ in the interval $\left(\underline{t}_{i-2}-\underline{t}_{i-1}\right)$. Minimizing the latter term requires that node $i+1$ receives no data in the interval $\left(t_{1}, t_{i-1}\right)$. Algorithm 1 satisfies that and hence establishes the inductive step.

\section{CONCLUSION}

This paper has considered the transmission scheduling issue in an upload-constrained peer-to-peer file distribution system. Under the assumptions that the network is static and that the file is infinitely divisible, an explicit transmission scheduling algorithm has been proposed which provably minimizes the average finish time for all peers. New inductive concepts like min-min times and novel techniques such as water-filling are used in obtaining the result.

There are a number of related directions in which to extend this work. First, it would be useful to investigate how the optimal results change when download constraints are introduced. Second, understanding the behavior of our optimal scheduling when nodes dynamically enter and leave upon completion [5], [24] would be necessary before its application in practice. Another interesting direction is to look at similar optimality results under peer-to-peer streaming [4], [6] context. Finally, this paper only gives the best possible centralized solution without any coding. Exploring corresponding distributed solutions or the effect of tools like network coding [2], [7], [10] can be potentially fruitful.

\section{ACKNOWLEDGEMENTS}

This research was performed under an appointment to the U.S. Department of Homeland Security (DHS) Scholarship and Fellowship Program, administered by the Oak Ridge Institute for Science and Education (ORISE) through an interagency agreement between the U.S. Department of Energy (DOE) and DHS. ORISE is managed by Oak Ridge Associated Universities (ORAU) under DOE contract number DEAC05-06OR23100. All opinions expressed in this paper are the author's and do not necessarily reflect the policies and views of DHS, DOE, or ORAU/ORISE.

\section{APPENDIX I}

\section{Proof OF THEOREM 1 WITH $1<M<N$}

Proof: The proof begins by establishing conditions for appropriate $\lambda$ values. It then finds the exact values of $\lambda$ and min-min times $\underline{t}_{1}, \ldots, \underline{t}_{M+1}$, and applies the water/heliumfilling concept to establish all remaining min-min times.
In order to achieve minimum $\underline{t}_{1} \ldots \underline{t}_{M}$, each node must relay whatever it receives from the server on $\left(0, \underline{t}_{M}\right)$ to nodes $i \in\{1, \ldots, M\}$. Thus, an upper bound on what each node can receive from the server on $\left(0, t_{M}\right)$ is

$$
\begin{array}{ll}
\lambda_{i} \leq \frac{C_{i}}{M-1} & \forall i \leq M \\
\lambda_{i} \leq \frac{C_{i}}{M} & \forall i>M
\end{array}
$$

Since Algorithm 1 keeps $\lambda_{i}$ values in these ranges, and relays all server streams to nodes $\{1, \ldots, M\}$, times $\underline{t}_{1}, \ldots, \underline{t}_{M}=|F| / C_{0}$ are minimized.

To establish a lower bound on $\underline{t}_{M+1}$, consider first how much data node $M+1$ can receive on $\left(0, t_{M}\right)$, from the server, nodes $\{1, \ldots, M\}$, itself, and nodes $\{M+2, \ldots, N\}$ :

$$
\begin{aligned}
\left|R_{0, M+1}\left(0, t_{M}\right)\right| & =\lambda_{M+1} t_{M} \\
\left|\bigcup_{i=1}^{M} R_{i, M+1}\left(0, t_{M}\right)\right| & \leq\left(\sum_{i=1}^{M} C_{i}-(M-1) \sum_{i=1}^{M} \lambda_{i}\right) t_{M} \\
\left|R_{M+1, M+1}\left(0, t_{M}\right)\right| & =0 \\
\bigcup_{i=M+2}^{N} R_{i, M+1}\left(0, t_{M}\right) \mid & \leq\left(\sum_{i=M+2}^{N} C_{i}-M \sum_{i=M+2}^{N} \lambda_{i}\right) t_{M}
\end{aligned}
$$

On $\left(t_{M}, t_{M+1}\right)$, each node $i \in\{0,1, \ldots, M\}$ could send to $M+1$ with rate $r_{i, M+1}(t)=C_{i}$, giving

$$
\left|\bigcup_{i=0}^{M} R_{i, M+1}\left(t_{M}, t_{M+1}\right)\right| \leq\left(t_{M+1}-t_{M}\right) \sum_{i=0}^{M} C_{i}
$$

The contribution $\bigcup_{i=M+2}^{N} R_{i, M+1}\left(t_{M}, t_{M+1}\right)$ of nodes $\{M+2, \ldots, N\}$ is limited both by their sum upload capacity, $\sum_{i=M+2}^{N} C_{i}$, and by the amount of information they received on $\left(0, t_{M}\right)$. Thus

$$
\begin{gathered}
\left|\bigcup_{i=M+2}^{N} R_{i, M+1}\left(t_{M}, t_{M+1}\right)\right| \leq \min \left(\sum_{i=M+2}^{N} C_{i}\left(t_{M+1}-t_{M}\right),\right. \\
\left.\quad \sum_{i=M+2}^{N} \lambda_{i} t_{1}-\left[\sum_{i=M+2}^{N} C_{i}-\sum_{i=M+2}^{N} \lambda_{i} M\right] t_{M}\right) .
\end{gathered}
$$

These combine to form the upper bound on the amount of information which can be received by node $M+1$ by time $t_{M+1}$ shown in (19). Also note that by definition, $F_{M+1}\left(t_{M+1}\right)=F$.

Considering each term of the min in (19) separately, and solving for $t_{M+1}$ yields two lower bounds on $t_{M+1}$ in terms of $\sum_{i=1}^{M} \lambda_{i}, \lambda_{M+1}$, and $\sum_{i=M+2}^{N} \lambda_{i}$.

$$
\begin{aligned}
& \text { When } \sum_{i=M+2}^{N} C_{i} t_{M+1} \leq \sum_{i=M+2}^{N}(M+1) \lambda_{i} t_{1}, \\
& \underline{t}_{M+1}\left(C-C_{M+1}\right) \geq \underline{t}_{M}(M-1) \sum_{i=1}^{M} \lambda_{i}-\underline{t}_{M} \lambda_{M+1} \\
& +\underline{t}_{M} C_{0}+\underline{t}_{M} M \sum_{i=M+2}^{N} \lambda_{i}+|F|
\end{aligned}
$$




$$
\begin{aligned}
\left|F_{M+1}\left(t_{M+1}\right)\right| \leq & \left(\sum_{i=1}^{M} C_{i}-(M-1) \sum_{i=1}^{M} \lambda_{i}\right) t_{M}-M \sum_{i=M+2}^{N} \lambda_{i} t_{M}+\lambda_{M+1} t_{M} \\
& +\left(t_{M+1}-t_{M}\right)\left(C_{0}+\sum_{i=1}^{M} C_{i}\right)+\min \left(\sum_{i=M+2}^{N} C_{i} t_{M+1}, \sum_{i=M+2}^{N}(M+1) \lambda_{i} t_{1}\right)
\end{aligned}
$$

and in the converse case

$$
\begin{aligned}
\underline{t}_{M+1} \sum_{i=0}^{M} C_{i} \geq & \underline{t}_{M}(M-1) \sum_{i=1}^{M} \lambda_{i}-\underline{t}_{M} \lambda_{M+1} \\
& +\underline{t}_{M} C_{0}-\underline{t}_{M} \sum_{i=M+2}^{N} \lambda_{i}+|F| .
\end{aligned}
$$

Note that in both cases, the lower bound is decreasing in $\lambda_{M+1}$, and so is minimized by maximizing $\lambda_{M+1}$ by setting

$$
\lambda_{M+1}=\frac{C_{M+1}}{M} .
$$

Since the bound given by (20) is increasing in $\sum_{i=M+2}^{N} \lambda_{i}$ and that given by (21) is decreasing, the min in (19) is minimized, for a given $C_{0}=\sum_{i=1}^{N} \lambda_{i}$, when the two bounds coincide. This gives the fundamental lower bound

$\underline{t}_{M+1} \geq \frac{\left(M^{2} C_{0}-M^{2} \lambda_{M+1}+2 M C_{0}-M \lambda_{M+1}+C_{0}\right)|F|}{C_{0}\left((M+1)\left(\sum_{i=0}^{M} C_{i}\right)+M \sum_{i=M+2}^{N} C_{i}\right)}$.

When $C_{0}>C_{0}^{*}$, the value of $\sum_{i=1}^{M} \lambda_{i}$ necessary to achieve this bound violates (15). In this case, the algorithm sets $\lambda_{i}, i<M$, to its upper bound of $C_{i} /(M-$ 1 ), and (18) becomes $\left|\bigcup_{i=M+2}^{N} R_{i, M+1}\left(t_{M}, t_{M+1}\right)\right|=$ $\sum_{i=M+2}^{N} C_{i} t_{M+1}$.

When $C_{0}>C_{0}^{*}$, nodes $i \in\{M+2, \ldots, N\}$ need not upload all of their information $F_{i}\left(t_{M}\right)$ to node $M$ to achieve the lower bound (18); it is sufficient that $\lambda_{i}, i \in\{M+$ $2, \ldots, N\}$, be large enough that $r_{i, M+2}(t)=C_{i}$ for all $t \in$ $\left(t_{M}, t_{M+1}\right)$. The LP (5) ensures that condition is met, while

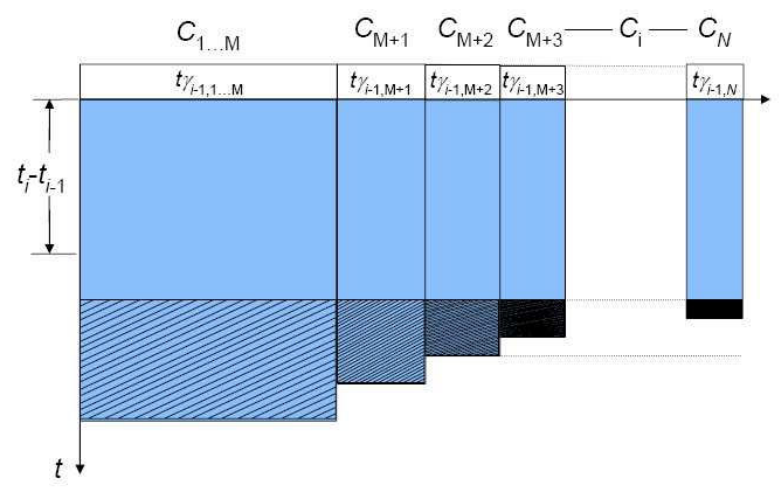

Fig. 5. A visual depiction of the waterfilling argument for the case when $1<M<N$ and $C_{0}>C_{0}^{*}$. Note the tiered structure of the columns for $i>M$. sequentially providing as much server capacity on $\left(0, t_{M}\right)$ as possible to nodes $M+2, \ldots, N$.

In either case, Algorithm 1 achieves the lower bound on $\underline{t}_{M+1}$ while maintaining $t_{1}, \ldots, t_{M}=|F| / C_{0}$.

Finally, we claim after $\underline{t}_{M+1}$, each node $i$ receives at rate $C-C_{i}$ on its finishing interval, and $C_{i-1}$ on the previous interval. To confirm, consider the fictional time interval when another node $k \notin\{1, \ldots, N\}$, needs to receive all information held by nodes $\{1, \ldots, N\}$ (i.e., it has no portion of the file). In this case, the amount of time it takes to transmit if all nodes have access to the entire file, $|F| / C$, is less than the amount of time it takes for any individual node to upload its assigned portion of the file, $\lambda_{i} t_{1} / C_{i}$.

Under Algorithm 1,

$$
\lambda_{N} \leq \lambda_{i}, \quad \forall i \in\{1, \ldots, N\},
$$

including in the case that $C_{0}>C_{0}^{*}$. To show that each node has enough information to transmit fully on any time interval, it is sufficient to show that

$$
\frac{\sum_{i=1}^{M} \lambda_{i}}{C_{0}+\sum_{i=1}^{N} C_{i}} \leq \frac{\lambda_{N}}{C_{N}}
$$

which can be reformed as

$$
\frac{C_{0}}{C} \leq \frac{\sum_{i=M+2}^{N} \lambda_{i}}{\sum_{i=M+2}^{N} C_{i}}
$$

and results in a bound of

$$
C_{0} \geq \frac{C_{M+1}\left(C-C_{0}\right)}{M C_{M+1}+\sum_{i=M+2}^{N} C_{i}} .
$$

This lower bound on $C_{0}$ for the condition to hold is strictly less than the lower bound due to the multiplicity constraint. Thus, full utilization is maintained for all time intervals prior to $\left(t_{N-1}, t_{N}\right)$ when following the suggested optimal scheme.

\section{REFERENCES}

[1] J. Chan, V. Li and K. Lui. Performance comparision of scheduling algorithms for peer-to-peer collaborative file distribution. IEEE Journal on Selected Areas in Communications, 25(1):146-154, January 2007

[2] P. Chou and Y. Wu. Network coding for the Internet and wireless networks. IEEE Signal Processing Magazine, 24(5):77-85, September 2007

[3] B. Fan, D. Chiu and J. Lui. The delicate tradeoffs in Bit Torrent-like file sharing protocol design. In Proccedings of IEEE ICNP, 2006.

[4] L. Gao, D. Towsley and J. Kurose. Efficient schemes for broadcasting popular videos. In Proceedings of ACM NOSSDAV, 1998.

[5] K. Gummadi, R. Gummadi, S. Gribble, S. Ratnasamy, S. Shenker and I. Stoica. The impact of DHT routing geometry on resilience and proximity. In Proceedings of ACM SIGCOMM, 2003. 
[6] X. Hei, C. Liang, Y. Liu and K. Ross. A measurement study of a large-scale P2P IPTV system. IEEE Transactions on Multimedia, 9(8):1672-1687, December 2007

[7] T. Ho, M. Mèdard and R. Koetter. An information-theoretic view of network management. IEEE Transactions on Information Theory, 51(4):1295-1312, April 2005

[8] R. Kumar and K. Ross. Peer-assisted file distribution: The minimal distribution time. In Proccedings of IEEE Workshop on Hot Topics in Web Systems and Technologies, 2006.

[9] J. Kurose and K. Ross. Computer Networking. Fourth edition, Addison Wesley, 2007.

[10] S. Li, R. Yeung and N. Cai Linear network coding IEEE Transactions on Information Theory, 49(2):371-381, February 2003

[11] M. Lingjun and K. Liu. Scheduling in P2P file distribution - On reducing the average distribution time. In Proceedings of Consumer Communications and Networking Conference, 2008.

[12] S. Liu, R. Shen, W. Jiang, J. Rexford and M. chiang. Performance bounds for peer-assisted live streaming. Proceedings of ACM SIGMETRICS, 2008.

[13] L. Massoulié and M. Vojnović. Coupon Replication Systems. IEEE/ACM Transactions on Networking, 16(3):603-616, June 2008

[14] M. Mehyar. Distributed Averaging and Efficient File Sharing on Peerto-Peer Networks. Doctoral Thesis, California Institute of Technology, 2006.

[15] M. Mehyar, G. WeiHsin, S. Low, M. Effros and T. Ho. Optimal strategies for efficient peer-to-Peer file sharing. Proceedings of IEEE ICASSP, 2007.

[16] J. Mundinger, R. Weber and G. Weiss. Analysis of peer-to-peer file dissemination amongst users of different upload capacities. $A C M$ SIGMETRICS Performance Evaluation Review, 34(2):5-6, September 2006.

[17] J. Mundinger, R. Weber and G. Weiss. Optimal scheduling of peerto-peer file dissemination. Journal of Scheduling, 11(2):1094-6136, April 2008.

[18] Q. Ou and D. Tsang. An optimal bandwidth allocation algorithm for file distribution network. In Proceedings of ChinaCom, 2007.

[19] J. Pouwelse, P. Garbacki, D. Epema, and H. Sips. The Bittorrent P2P file-sharing system: Measurements and analysis. Proceedings of 4th International Workshop on Peer-to-Peer Systems, 2005.

[20] D. Qiu, and R. Srikant. Modeling and performance analysis of BitTorrent-like peer-to-peer networks. Proceedings of ACM Sigcomm, 2004.

[21] S. Sanghavi, B. Hajek and L. Massouliè. Gossiping with multiple messages. IEEE Transactions on Information Theory, 53(12):46404654, December 2007

[22] I. Stoica, R. Morris, D. Karger, M. Kaashoek and H. Balakrishnan. A scalable peer-to-peer lookup service for Internet applications. Proceedings of ACM SIGCOMM, 2001.

[23] X. Yang and G. De Veciana. Service capacity of peer to peer networks Proceedings of IEEE Infocom, 2004.

[24] Z. Yao, D. Leonard, X. Wang and D. Loguinov. Modeling heterogeneous user churn and local resilience of unstructured P2P networks. In Proceedings of IEEE ICNP, 2006.

[25] X. Zheng, C. Cho and Y. Xia Optimal peer-to-peer techniques for massive content distribution. In Proceedings of IEEE Infocom, 2008. 